\title{
Terahertz Laser Vibration-Rotation Tunneling Spectroscopy of the Water Tetramer
}

\author{
Jeff D. Cruzan, ${ }^{\dagger}$ Mark R. Viant, Mac G. Brown, and Richard J. Saykally* \\ Department of Chemistry, University of California at Berkeley, Berkeley, California 94720
}

Received: March 3, 1997; In Final Form: June 20, $1997^{\circledR}$

\begin{abstract}
A vibration-rotation-tunneling (VRT) spectrum of $\left(\mathrm{H}_{2} \mathrm{O}\right)_{4}$ has been recorded near $2.04 \mathrm{THz}\left(67.9 \mathrm{~cm}^{-1}\right)$. The band origin of this $\Delta K=0$ symmetric rotor spectrum is shifted by $0.1 \mathrm{~cm}^{-1}$ to the blue of that of a $\left(\mathrm{D}_{2} \mathrm{O}\right)_{4}$ band reported previously [Science, 1996, 271, 59]. Similar to that spectrum, each transition in the $\left(\mathrm{H}_{2} \mathrm{O}\right)_{4}$ spectrum exhibits a regular doublet splitting. The $\left(\mathrm{D}_{2} \mathrm{O}\right)_{4}$ and $\left(\mathrm{H}_{2} \mathrm{O}\right)_{4}$ doublet spacings, 5.6 and 2260 $\mathrm{MHz}$, respectively, are constant as a function of rotation, indicative of degenerate tunneling splittings. They have been rationalized in terms of a concerted flipping motion of the $\mathrm{H}$ atoms that do not participate in hydrogen bonding in the $S_{4}$ equlibrium structure, analogous to ammonia inversion. Both bands have been assigned to the lowest $\mathrm{A}_{\mathrm{g}} \rightarrow \mathrm{B}_{\mathrm{g}}$ intermolecular vibration in the molecular symmetry group $C_{4 h}(\mathrm{M})$. Additional $K=2$ splittings, which increase nonlinearly with increasing $J$, were found in both $\mathrm{D}_{2} \mathrm{O}$ and $\mathrm{H}_{2} \mathrm{O}$ tetramer spectra. Evidence for the bifurcation tunneling motion observed in the water trimer VRT data, but not in evidence for $\left(\mathrm{D}_{2} \mathrm{O}\right)_{4}$, was found in the $\left(\mathrm{H}_{2} \mathrm{O}\right)_{4}$ spectrum in the form of broadened lines probably containing unresolved bifurcation tunneling splittings. Structural estimates from the data indicate that the interoxygen separations in both $\left(\mathrm{D}_{2} \mathrm{O}\right)_{4}$ and $\left(\mathrm{H}_{2} \mathrm{O}\right)_{4}$ are consistent with an exponential contraction in that parameter as a function of increasing cluster size.
\end{abstract}

\section{Introduction}

A series of recent high-resolution studies of gas-phase water clusters as large as the hexamer in the terahertz region of the spectrum have produced a wealth of new experimental data to test theoretical models of water clusters and bulk phase water. ${ }^{1}$ A particular goal of these studies has been to quantify the nature and consequences of many-body forces in determining the properties of both clusters and the bulk phases. ${ }^{2}$ Experimental studies of the gas-phase water dimer ${ }^{3}$ and trimer $^{4-11}$ have now produced an extensive body of data that will eventually lead to complete, detailed representations of the intermolecular potential energy surfaces (IPSs) of those systems. Determination of a water dimer IPS accurate to the precision of the terahertz vibration-rotation-tunneling (VRT) spectroscopic measurements $\left(<0.001 \mathrm{~cm}^{-1}\right)$, will provide a fundamental basis for developing a more complete effective potential for use in simulations of condensed phases. The requisite three-body terms can be extracted from the trimer IPS.

$A b$ initio calculations at the highest practical levels of electron correlation [MP4, CCSD(T)] have predicted both a structural contraction and an increase in per-monomer binding energies of cyclic water clusters as a function of increasing size for $\left(\mathrm{H}_{2} \mathrm{O}\right)_{n}, n=2-6$, as a result of many-body interactions. ${ }^{12-14}$ Most studies agree that the many-body expansion of water interactions in terms of two-, three-, $\ldots, n$-body forces, is rapidly convergent, with four body forces accounting for on the order of $1 \%$ of the total stabilization energy of the tetramer and pentamer. ${ }^{12-21}$ Xantheas' recent MP2 calculations predicted that three-body forces contribute 18 and $26 \%$ of the total stabilization energy of the water trimer and tetramer, respectively. ${ }^{12,17-19}$ The reason for the increase is a convolution of several factors, including the anisotropies of both the pairwise additive forces (dominated by electrostatic interactions) and of the many-body components. Chałasiński and co-workers calculated the ani-

\footnotetext{
$\dagger$ Present address: Department of Molecular and Cellular Biology, Harvard University, 7 Divinity Ave., Cambridge, MA 02138.

* To whom correspondence should be addressed.

${ }^{\otimes}$ Abstract published in Advance ACS Abstracts, October 1, 1997.
}

sotropy of additive and nonadditive forces in the water trimer as a function of the $\mathrm{H}$-bonding angle $\delta(\mathrm{O}-\mathrm{H} \cdots \mathrm{O})$, which is $180^{\circ}$ for a linear $\mathrm{H}$ bond. ${ }^{22,23}$ In agreement with much early work, ${ }^{24}$ they showed that the additive forces predominantly cause the preference for near-linear H-bonds. Belford and Campbell pointed out that proper consideration of those additive forces, including two-body dispersion, which is the most problematic aspect of intermolecular interactions in ab initio modeling, is crucial in order to properly predict the many-body contributions, which are highly anisotropic and range sensitive. ${ }^{25}$ Many studies, particularly those of Clementi and co-workers, ${ }^{26-30}$ have shown that modification of effective pair potentials by inclusion of three- and four-body interactions is necessary in order to accurately model liquid and solid water properties, including geometric distribution functions, and thermodynamic properties such as the anomalous heat capacity. While simple pair potentials can give reasonable results for the structures of water clusters smaller than the pentamer, larger clusters require either high levels of ab initio theory or inclusion of many-body terms in effective potentials in order to correctly establish the global minima of larger clusters. ${ }^{31-33}$ Ohmine's review of hydrogen bond network rearrangement (HBNR) dynamics in liquid water provides a good overview of the manifestations of H-bond cooperativity in the dynamics of liquid water ${ }^{34}$

The consensus of most ab initio calculations ${ }^{12,31,35-41}$ and other theoretical studies ${ }^{39,42,43}$ is that the equilibrium structure of the water tetramer is the one shown in Figure 1. It is analogous to the water trimer and pentamer in that each monomer acts as both a single H-bond donor and acceptor, with one proton (or deuteron) $\mathrm{H}$-bonded in the ring framework and one relatively free to rotate torsionally about (or approximately about) the $\mathrm{H}$-bonded $\mathrm{O}-\mathrm{H}$ axis. But unlike the trimer and pentamer, which have chiral global minimum structures $\left(C_{1}\right.$ symmetry), the tetramer has $S_{4}$ symmetry and is therefore a symmetric top even in the rigid molecule limit.

Terahertz (or far-IR) VRT spectroscopy directly probes of the intermolecular vibrational eigenstates characterizing the H-bonds in water clusters. ${ }^{1,44}$ FIR-VRT studies of cyclic 


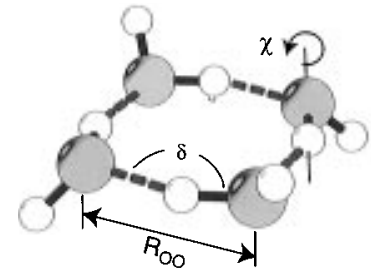

Figure 1. The consensus of theoretical calculations is that the equilibrium structure of the water tetramer is the cyclic one shown here, corresponding to a symmetric top with $S_{4}$ point group symmetry.

tetramers ${ }^{45,46}$ and pentamers, ${ }^{47,48}$ and a three-dimensional cage form of the hexamer, ${ }^{49}$ have been reported, along with earlier results for the dimer and trimer, allowing useful comparisons to be drawn between theory and experiment. One consequence of the intermolecular dynamics observed in the trimer and pentamer VRT data is that those spectra are rigorously those of symmetric top molecules, in apparent contradiction to the calculated global minimum structures. However, it has been shown that the cluster structures are averaged over many periods of the flipping motion on the time scale of molecular rotation, leading to effective $C_{3 h}$ or $C_{5 h}$ symmetry and thus producing symmetric top spectra. ${ }^{6,50-53}$ Although it seems reasonable, by interpolation, to suspect that the flipping coordinates should be active in the analogous cyclic tetramer, no a priori information on the flipping coordinates is evident in the rotational constants because the global minimum structure is already a symmetric top, i.e., the observation of a symmetric rotor spectrum does not require flipping to occur. This fact has complicated the analyses of the tetramer VRT data somewhat.

A second dynamical effect observed in the water trimer VRT spectra but not in the tetramer or pentamer data obtained thus far, is due to "bifurcation tunneling" (or "donor tunneling"), exchange of the H-bonded and free protons of one monomer via a pathway that involves a bifurcated H-bond. ${ }^{51,52,54}$ Bifurcation tunneling has been shown to give rise to quartet splittings in each transition in the trimer VRT spectra, which are a now familiar spectroscopic fingerprint of those clusters. ${ }^{6,51,52}$ Several theoretical studies have predicted that the per monomer binding energy increases logarithmically for the series beginning with the dimer and ending with the (theoretically cyclic) hexamer, for which convergence of the per monomer stabilization energy to the neighborhood of $-6.5 \mathrm{kcal} / \mathrm{mol}$ is generally observed. ${ }^{12,18,19,43}$ The same calculations predict an average $\mathrm{H}$-bond energy of $-4.5 \mathrm{kcal} / \mathrm{mol}$ for the dimer. The larger structures are therefore more rigidly bound than the dimer and trimer, possibly serving to quench some of the otherwise observable rearrangement dynamics.

Up to this report, only the perdeuterated species of the water tetramer and pentamer had been observed. Here we describe observation and analysis of a $\left(\mathrm{H}_{2} \mathrm{O}\right)_{4}$ spectrum recorded near $67.9 \mathrm{~cm}^{-1}$, less than $0.1 \mathrm{~cm}^{-1}$ from the band origin of the first $\left(\mathrm{D}_{2} \mathrm{O}\right)_{4}$ absorption spectrum. ${ }^{45,46}$ Some key questions left open in that initial study are further illuminated by the new data. In particular, the interpretation of the dominant spectral (doublet) splittings reported in refs 45 and 46 is reinforced by the new data and by theoretical studies stimulated by that work. Using the diffusion quantum Monte Carlo (DQMC) technique that they have applied to the water dimer and trimer, ${ }^{20,53,55-57}$ Gregory and Clary ${ }^{58}$ have now calculated tunneling splittings in $\left(\mathrm{H}_{2} \mathrm{O}\right)_{4}$ and $\left(\mathrm{D}_{2} \mathrm{O}\right)_{4}$, finding evidence in support of the hypothesis ${ }^{45,46}$ that the observed doublet splittings arise from a concerted tunneling motion similar to the inversion coordinate in ammonia. ${ }^{59}$ In addition, the ab initio studies of Schütz and coworkers $^{38}$ and of Xantheas and Dunning, ${ }^{12}$ have provided a tentative vibrational assignment of both VRT bands. Neverthe- less, some puzzling features that must reflect the complicated cluster dynamics are evident in both data sets and remain difficult to rationalize.

\section{Experimental and Results}

The Berkely far-IR spectrometers have been discussed in detail elsewhere. ${ }^{60}$ The details of the present experiment are essentially the same as those described in ref 46. Water clusters were formed by expanding $\mathrm{H}_{2} \mathrm{O}$ (distilled tap water), seeded in a rare gas, into a vacuum chamber through the $10 \mathrm{~cm}$ long pulsed, slit-orifice supersonic nozzle beam source designed and described by Liu et al. ${ }^{61}$ Frequency-modulated $(50 \mathrm{kHz})$ tunable laser sidebands probed the expansion between 0.5 and $2 \mathrm{~cm}$ downstream of the nozzle orifice before being focused on a mechanically stressed Ge:Ga photoconductive detector. Use of a Herriott multipass cell ensured effective absorption path lengths of over $2 \mathrm{~m} .{ }^{62}$ A gated lock-in detection scheme was used to recover the signal. Essentially, the frequency modulated laser sidebands were demodulated by a fast digital lock-in amplifier (Stanford SR810) and fed into the input of a Tektronix TDS-320 digital storage oscilloscope. Typically, 32 or 64 nozzle pulses were averaged per frequency interval by the oscilloscope before direct downloading of the digitized, averaged waveform to a $486 \mathrm{PC}$ computer for integration of the $2 \mathrm{~ms}$ wide signal window. The peak signal-to-noise ratio of the present band obtained in this manner was 20:1 for the strongest Q-branch lines.

The $\left(\mathrm{H}_{2} \mathrm{O}\right)_{4}$ spectrum, a $\Delta K=0$ band of an oblate symmetric rotor, was measured in the same region of the far-IR spectrum as the previously measured $\left(\mathrm{D}_{2} \mathrm{O}\right)_{4}$ band, ${ }^{45,46}$ near $67.9 \mathrm{~cm}^{-1}$. The identity of the spectral carrier was confirmed by several tests. First, signals were observed in nozzle beam expansions of $\sim 1 \% \mathrm{H}_{2} \mathrm{O}$ in $\mathrm{Ar}$, $\mathrm{Ne}$ and $\mathrm{Kr}$, therefore clusters containing any of those rare gas components were ruled out. Second, as described in refs 45 and 46 , and by Pugliano, ${ }^{8}$ isotopic substitution experiments were performed in order to determine the number of water molecules in the cluster. The essential feature of these analyses, which have been used to confirm the identity of several water clusters, is that the concentration of $\mathrm{H}_{2} \mathrm{O}$ or $\mathrm{D}_{2} \mathrm{O}$ available for clustering can be varied in a controlled manner by preparing statistical mixtures of $\mathrm{H}_{2} \mathrm{O}$ and $\mathrm{D}_{2} \mathrm{O}$. If the intensity of a given signal from a cluster $\left(\mathrm{H}_{2} \mathrm{O}\right)_{n}$ is arbitrarily fixed to unity, the intensity of the same signal recorded using a mixture of $\mathrm{H}_{2} \mathrm{O}$ and $\mathrm{D}_{2} \mathrm{O}$, where $\chi_{\mathrm{H}_{2} \mathrm{O}}$ and $\chi_{\mathrm{D}_{2} \mathrm{O}}$ are the corresponding mole fractions, is given to a good approximation by

$$
\ln \left(I_{\text {rel }}\right)=2 n \ln \left(\chi_{\mathrm{H}_{2} \mathrm{O}}\right)
$$

where $I_{\text {rel }}$ is the intensity relative to the pure signal. Formula 1 arises from the recognition that any mixture of $\mathrm{H}_{2} \mathrm{O}$ and $\mathrm{D}_{2} \mathrm{O}$ will rapidly randomize to a statistical mixture of $\mathrm{H}_{2} \mathrm{O}, \mathrm{D}_{2} \mathrm{O}$ and HOD. A linear least-squares fit of formula 1 to pairs of concentrations and relative intensities can be used to determine $n$, the number of $\mathrm{H}_{2} \mathrm{O}$ (this case) monomers in the cluster, as the slope of the graph. The principal uncertainty in these tests is usually in the intensity measurements and is due to instrumental fluctuations, for example, laser frequency drift. Implicit in these experiments is the assumption that there is no significant isotope effect in the cluster formation process.

Interestingly, a study by Kay and Castleman ${ }^{63}$ casts some aspersion on that assumption and is worth addressing. In an RRKM kinetic model of cluster formation in free jet expansions, they showed that the higher density of states expected in deuterated clusters leads to preferential loss of $\mathrm{H}_{2} \mathrm{O}$ over heavier 


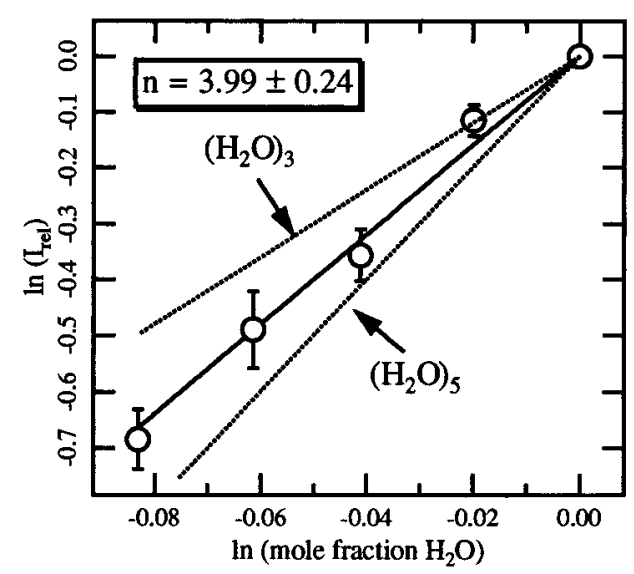

Figure 2. Results of the isotopic substitution experiment performed to determine the number of water monomers in the $\left(\mathrm{H}_{2} \mathrm{O}\right)_{4}$ VRT spectrum. The broken lines indicate expected results for $\mathrm{H}_{2} \mathrm{O}$ trimer and pentamer. The results confirm that the cluster contains four monomers. The isotopic enrichment effect discussed in the text would not be expected to contribute in excess of the error bars.

monomers in processes whereby collisionally excited larger clusters relax to form more stable forms by ejection of a monomer. They combined their theoretical efforts with experimental data that confirmed the results. In a mass detection experiment, they observed isotopic enrichment of the deuterated species of as much as $30 \%$, measured as the deviation from the result expected in the pure statistical case. These effects, however, were measured in $\mathrm{H}_{2} \mathrm{O} / \mathrm{D}_{2} \mathrm{O}$ mixtures near $50 \%$, wherein HOD is the dominant species. In the isotopic substitution experiments described in the present work, mixtures of at most $20 \%$ impurity are used. This must be so because the probability of obtaining accurate relative intensities diminishes as the signals approach the noise limit. For example, in a $85 \%$ $\mathrm{H}_{2} \mathrm{O}+15 \% \mathrm{D}_{2} \mathrm{O}$ mixture, signals of $\left(\mathrm{H}_{2} \mathrm{O}\right)_{n}$ for $n=3,4,5$, and 6 diminish (from a normalized strength of 1 ) to $0.37,0.27$, 0.19 , and 0.14 , respectively. An $85 \%$ mixture is unacceptable for pentamer and hexamer tests unless the original signals are very strong. Higher $\mathrm{H}_{2} \mathrm{O} / \mathrm{D}_{2} \mathrm{O}$ ratios, with $\chi_{\mathrm{H}_{2} \mathrm{O}}=90-99 \%$, are more generally used. For these ratios, the results of Kay and Castleman are less important because the majority water species in the expansion is the one of interest. Kay and Castleman also carefully measured the relative concentrations of $\mathrm{H}_{2} \mathrm{O}$ and $\mathrm{D}_{2} \mathrm{O}$ at the end of their experiments and found no preferential vaporization of either species, indicating that the small difference in vapor pressures of the isotopomers should not lead to measurable isotope effects. It is reasonable, therefore, to conclude that the isotopic substitution experiments are a reliable method for determining the number of monomers in a water cluster as long as the mixtures used are near unity in the concentration of the monomer of interest. Additionally, if a significant isotope effect were at work at the impurity concentrations used in the VRT experiments, it would be manifested primarily in the intercept rather than in the slope of graphs of formula 1, and the number of hydrogens in a given cluster is extracted from the latter quantity.

The results of the isotope analysis performed on the $\left(\mathrm{H}_{2} \mathrm{O}\right)_{4}$ data are shown in Figure 2. The experiment yielded $n=4 \pm$ $0.23(1 \sigma)$. The final-and most rigorous - test of the identity of the chromophore was in the rotational parameters obtained from analysis of the data (with a spectral resolution on the order of $1 \mathrm{MHz}$, the VRT experiments can easily distinguish between tetramers with even a single $\mathrm{H} \leftrightarrow \mathrm{D}$ substitution), and in the similarity of the primary doublet splitting patterns to those observed in the $\left(\mathrm{D}_{2} \mathrm{O}\right)_{4}$ spectrum. Furthermore, the additional



Figure 3. The observed VRT transitions of the $67.9 \mathrm{~cm}^{-1}\left(\mathrm{H}_{2} \mathrm{O}\right)_{4}$ spectrum shown as a stick spectrum with calculated intensities. The two subbands are easily recognized as a doubling of every cluster of lines characteristic of a $\Delta K=0$ symmetric top spectrum. The lower spectrum is a blowup of the central Q-branch region.

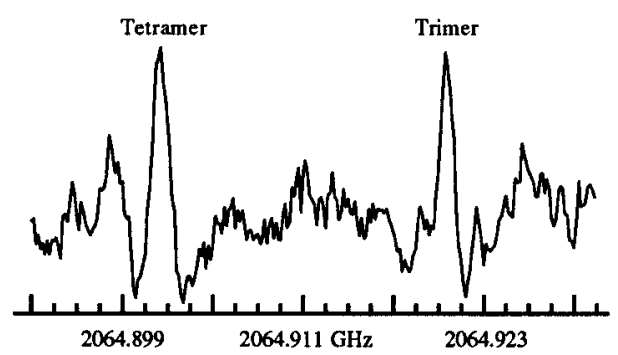

Figure 4. This scan shows the $4_{0} \leftarrow 3_{0}$ transition of the $\left(\mathrm{H}_{2} \mathrm{O}\right)_{4}$ spectrum near a transition of a neighboring $\left(\mathrm{H}_{2} \mathrm{O}\right)_{3}$ band. The $\left(\mathrm{H}_{2} \mathrm{O}\right)_{4}$ line is roughly 1.5 times broader than the trimer line, which exhibits the normal Doppler broadened $\sim 1 \mathrm{MHz}$ line width at $70 \mathrm{~cm}^{-1}$. All of the $\left(\mathrm{H}_{2} \mathrm{O}\right)_{4}$ transitions were broadened by this amount (see Figure 5).

$K=2$ splittings observed in both the $\left(\mathrm{D}_{2} \mathrm{O}\right)_{4}$ and $\left(\mathrm{H}_{2} \mathrm{O}\right)_{4}$ spectra serve to "fingerprint" the tetramer data.

In total, 110 rotation-vibration lines due to $\left(\mathrm{H}_{2} \mathrm{O}\right)_{4}$, which at first appeared to describe two distinct $\Delta K=0$ symmetric rotor VRT spectra, were observed and assigned. They are illustrated in Figure 3. A typical $50 \mathrm{MHz}$ scan is reproduced in Figure 4. Both subbands could be described by a simple oblate symmetric rotor energy level expression including small quartic centrifugal distortion parameters. In light of the observation of doublet splittings of $5.6 \pm 0.01 \mathrm{MHz}$ in the $\left(\mathrm{D}_{2} \mathrm{O}\right)_{4}$ spectrum, the differences between transitions with the same $J$ and $K$ assignment were compared. From the experimental data listed in Table 1, it is easy to see that the differences between lines with the same $J_{K^{\prime}}^{\prime} \leftarrow J^{\prime \prime}{ }_{K^{\prime \prime}}$ assignment are centered around $2.26 \mathrm{GHz}$, constant to within the $3-4 \mathrm{MHz}$ uncertainty of the measurements (The uncertainty is somewhat greater than the resolution because it arises from long-term frequency drift of the laser). The remainder of the analysis was predicated on the assumption that the $2.26 \mathrm{GHz}$ difference, although quite large, was the analogue of the $5.6 \mathrm{MHz}$ splitting observed in the $\left(\mathrm{D}_{2} \mathrm{O}\right)_{4}$ spectrum. The modified symmetric rotor energy level expression

$$
\begin{array}{r}
E_{J, K}^{ \pm}=v+B J(J+1)+(C-B) K^{2}-D_{J} J^{2}(J+1)^{2}- \\
D_{J K} J(J+1) K^{2} \pm \alpha / 2
\end{array}
$$

(where $B$ and $C$ are the unique symmetric rotor rotational constants, $D_{J}$ and $D_{J K}$ are quartic centrifugal distortion parameters, $\alpha$ is the doublet splitting, and \pm refer to the upper and lower subbands, respectively) was used to fit the data in Table 1. This analysis differs somewhat from that performed on the 
TABLE 1: $\left(\mathrm{H}_{2} \mathrm{O}\right)_{4}$ Transitions $^{a}$

\begin{tabular}{|c|c|c|c|c|c|}
\hline \multirow[b]{2}{*}{$J_{K^{\prime}}^{\prime} \leftarrow J^{\prime \prime}{ }_{K^{\prime \prime}}$} & \multicolumn{2}{|c|}{ upper band } & \multicolumn{2}{|c|}{ lower band } & \multirow[b]{2}{*}{$|\Delta|$} \\
\hline & freq/MHz & res $^{b}$ & freq/MHz & $\operatorname{res}^{b}$ & \\
\hline $4_{0} \leftarrow 5_{0}$ & 2001770 & -0.7 & 1999512 & 1.9 & 2258 \\
\hline $4_{1} \leftarrow 5$ & 2001748 & -1.5 & 1999491 & 2.0 & 2257 \\
\hline $4_{2} \leftarrow 5_{2}$ & 2001685 & -1.0 & 1999427 & 2.3 & 2258 \\
\hline $4_{3} \leftarrow 5_{3}$ & 2001573 & -5.9 & 1999321 & 2.3 & 2253 \\
\hline $4_{4} \leftarrow 5_{4}$ & 2001432 & 1.0 & 1999172 & 2.7 & 2259 \\
\hline $3_{0} \leftarrow 4_{0}$ & 2008653 & 0.4 & 2006391 & -1.6 & 2263 \\
\hline $3_{1} \leftarrow 4_{1}$ & 2008632 & 0.4 & 2006373 & 1.8 & 2259 \\
\hline $3_{2} \leftarrow 4_{2}$ & 2008568 & -0.4 & & & \\
\hline $3_{3} \leftarrow 4_{3}$ & 2008463 & 0.6 & & & \\
\hline $20 \leftarrow 30$ & 2015572 & 1.2 & 2013312 & 2.8 & 2259 \\
\hline $2_{1} \leftarrow 3_{1}$ & 2015550 & 0.8 & 2013291 & 2.8 & 2259 \\
\hline $2_{2} \leftarrow 3_{2}$ & 2015487 & 0.8 & 2013222 & -2.7 & 2264 \\
\hline $2_{2} \leftarrow 2_{2}$ & 2036544 & -1.2 & 2034286 & 2.0 & 2258 \\
\hline $3_{3} \leftarrow 3_{3}$ & 2036540 & -1.3 & 2034282 & 1.6 & 2258 \\
\hline $4_{4} \leftarrow 4_{4}$ & 2036528 & -0.7 & 2034269 & 1.1 & 2259 \\
\hline $5_{5} \leftarrow 5_{5}$ & 2036508 & -0.3 & 2034246 & -1.2 & 2262 \\
\hline $6_{6} \leftarrow 6_{6}$ & 2036480 & -0.1 & 2034217 & -1.8 & 2263 \\
\hline $7_{7} \leftarrow 7_{7}$ & 2036443 & 0.3 & 2034179 & -2.8 & 2264 \\
\hline $8_{8} \leftarrow 8_{8}$ & 2036398 & 0.9 & 2034133 & -3.9 & 2266 \\
\hline $9_{9} \leftarrow 9_{9}$ & 2036345 & 1.3 & 2034078 & -4.9 & 2267 \\
\hline $10_{10} \leftarrow 10_{10}$ & 2036284 & 2.3 & 2034015 & -5.9 & 2269 \\
\hline $11_{11} \leftarrow 11_{11}$ & 2036214 & 3.1 & 2033943 & -7.5 & 2271 \\
\hline $12_{12} \leftarrow 12_{12}$ & 2036137 & 4.0 & 2033863 & -9.4 & 2274 \\
\hline $13_{13} \leftarrow 13_{13}$ & 2036050 & 4.5 & & & \\
\hline $14_{14} \leftarrow 14_{14}$ & 2035956 & 5.3 & & & \\
\hline $3_{2} \leftarrow 3_{2}$ & 2036652 & 6.3 & 2034385 & -0.2 & 2267 \\
\hline $4_{3} \leftarrow 4_{3}$ & 2036674 & -1.5 & 2034415 & 0.1 & 2259 \\
\hline $5_{4} \leftarrow 5_{4}$ & 2036696 & -1.4 & 2034437 & 0.3 & 2259 \\
\hline $6_{5} \leftarrow 6_{5}$ & 2036709 & -0.9 & 2034449 & -0.4 & 2260 \\
\hline $7_{6} \leftarrow 7_{6}$ & 2036715 & -0.4 & 2034453 & -1.3 & 2262 \\
\hline $8_{7} \leftarrow 8_{7}$ & 2036712 & 0.0 & & & \\
\hline $9_{8} \leftarrow 9_{8}$ & 2036700 & 0.3 & & & \\
\hline $10_{9} \leftarrow 10_{9}$ & 2036680 & 0.0 & & & \\
\hline $11_{10} \leftarrow 11_{10}$ & 2036652 & 0.7 & & & \\
\hline $12_{11} \leftarrow 12_{11}$ & 2036617 & 2.0 & & & \\
\hline $3_{1} \leftarrow 3_{1}$ & 2036709 & 0.5 & 2034449 & 1.0 & 2260 \\
\hline $5_{3} \leftarrow 5_{3}$ & 2036842 & -2.1 & & & \\
\hline $6_{4} \leftarrow 6_{4}$ & 2036897 & -2.0 & 2034638 & 0.1 & 2259 \\
\hline $7_{5} \leftarrow 7_{5}$ & 2036945 & -1.0 & 2034685 & -0.5 & 2260 \\
\hline $8_{6} \leftarrow 8_{6}$ & 2036983 & -1.1 & & & \\
\hline $9_{7} \leftarrow 9_{7}$ & 2037014 & -0.8 & & & \\
\hline $10_{8} \leftarrow 10_{8}$ & 2037036 & -0.6 & & & \\
\hline $11_{9} \leftarrow 11_{9}$ & 2037050 & -0.1 & & & \\
\hline $12_{10} \leftarrow 12_{10}$ & 2037056 & 0.7 & & & \\
\hline $13_{11} \leftarrow 13_{11}$ & 2037053 & 1.0 & & & \\
\hline $14_{12} \leftarrow 14_{12}$ & 2037043 & 2.2 & & & \\
\hline $4_{1} \leftarrow 4_{1}$ & 2036842 & -1.7 & & & \\
\hline $5_{2} \leftarrow 5_{2}$ & 2036945 & -4.2 & & & \\
\hline $6_{3} \leftarrow 6_{3}$ & 2037043 & -2.4 & & & \\
\hline $4_{0} \leftarrow 3_{0}$ & 2064941 & -0.1 & 2062684 & 3.3 & 2257 \\
\hline $4_{1} \leftarrow 3_{1}$ & 2064920 & -0.1 & 2062661 & 1.7 & 2259 \\
\hline $4_{2} \leftarrow 3_{2}$ & 2064858 & 0.0 & 2062599 & 1.3 & 2260 \\
\hline $4_{3} \leftarrow 3_{3}$ & 2064755 & 0.0 & 2062495 & 1.2 & 2260 \\
\hline $5_{0} \leftarrow 4_{0}$ & 2072126 & -0.7 & 2069864 & -1.9 & 2262 \\
\hline $5_{1} \leftarrow 4_{1}$ & 2072105 & -0.5 & 2069846 & 0.6 & 2260 \\
\hline $5_{2} \leftarrow 4_{2}$ & 2072043 & -0.3 & 2069784 & 1.5 & 2259 \\
\hline $5_{3} \leftarrow 4_{3}$ & 2071940 & -0.5 & 2069679 & -0.4 & 2261 \\
\hline $5_{4} \leftarrow 4_{4}$ & 2071796 & 0.2 & 2069537 & 1.9 & 2259 \\
\hline $6_{0} \leftarrow 5_{0}$ & 2079343 & -0.8 & 2077085 & 1.8 & 2258 \\
\hline $6_{1} \leftarrow 5_{1}$ & 2079323 & -0.6 & 2077064 & 1.7 & 2259 \\
\hline $6_{2} \leftarrow 5_{2}$ & 2079261 & -0.2 & 2077002 & 1.3 & 2259 \\
\hline $6_{3} \leftarrow 5_{3}$ & 2079159 & 0.5 & 2076898 & 0.9 & 2260 \\
\hline \multirow[t]{2}{*}{$6_{4} \leftarrow 5_{4}$} & 2079015 & 0.7 & 2076754 & 0.8 & 2261 \\
\hline & & & 2076568 & 0.5 & \\
\hline $7_{0} \leftarrow 6_{0}$ & 2086592 & -1.4 & 2084333 & 0.6 & 2259 \\
\hline $7_{1} \leftarrow 6_{1}$ & 2086571 & -1.3 & 2084312 & 0.5 & 2259 \\
\hline $7_{2} \leftarrow 6_{2}$ & 2086510 & -0.8 & 2084251 & 0.7 & 2259 \\
\hline $7_{3} \leftarrow 6_{3}$ & 2086408 & -0.5 & 2084147 & 0.2 & 2260 \\
\hline $7_{4} \leftarrow 6_{4}$ & 2086264 & -0.5 & 2084003 & 0.2 & 2260 \\
\hline $7_{5} \leftarrow 6_{5}$ & 2086079 & 0.1 & 2083818 & -0.4 & 2261 \\
\hline $7_{6} \leftarrow 6_{6}$ & 2085853 & 0.2 & 2083591 & -1.1 & 2262 \\
\hline
\end{tabular}

${ }^{a}$ In megahertz. ${ }^{b}$ Res $=$ residuals. Residuals are observed minus calculated frequencies from the fit of eq 2 to the data.
TABLE 2: Rotational Parameters (MHz) Determined from a Fit of Equation 2 to the $\left(\mathrm{H}_{2} \mathrm{O}\right)_{4}$ Data $^{a}$

\begin{tabular}{cll}
\hline parameter & \multicolumn{1}{c}{$\left(\mathrm{H}_{2} \mathrm{O}\right)_{4}$} & \multicolumn{1}{c}{$\left(\mathrm{D}_{2} \mathrm{O}\right)_{4}$} \\
\hline$\nu_{0}$ & $2035397.57(40)$ & $2032688.38(33)$ \\
$B^{\prime \prime}$ & $3509.994(79)$ & $3079.512(36)$ \\
$D_{J}^{\prime \prime}$ & $0.0123(13)$ & $0.0089(04)$ \\
$D_{J K}^{\prime \prime}$ & $-0.0287(31)$ & $-0.0177(07)$ \\
$B^{\prime}$ & $3526.817(83)$ & $3091.726(31)$ \\
$D_{J}^{\prime}$ & $\left(=D_{J}^{\prime \prime}\right)$ & $0.0092(03)$ \\
$D_{J K}^{\prime}$ & $\left(=D_{J K}^{\prime \prime}\right)$ & $-0.0181(06)$ \\
$\Delta \mathrm{C}$ & $-4.1465(53)$ & $-3.4771(67)$ \\
$\alpha$ & $2260.83(43)$ & $5.60(01)$
\end{tabular}

${ }^{a}$ The centrifugal distortion constants $D_{J}$ and $D_{J K}$ were constrained to be equal in the upper and lower states in the $\left(\mathrm{H}_{2} \mathrm{O}\right)_{4}$ fit due to large correlations when they were varied independently. $\alpha$ is the average doublet tunneling splitting. The analogous $\left(\mathrm{D}_{2} \mathrm{O}\right)_{4}$ parameters are included for comparison.

TABLE 3: Comparison of the $K=2$ Splittings (MHz) Observed in the $\left(\mathrm{D}_{2} \mathrm{O}\right)_{4}$ and $\left(\mathrm{H}_{2} \mathrm{O}\right)_{4}$ Bands $^{a}$

\begin{tabular}{ccc}
\hline & \multicolumn{2}{c}{ splitting/MHz } \\
\cline { 2 - 3 } transition & $\left(\mathrm{D}_{2} \mathrm{O}\right)_{4}$ & $\left(\mathrm{H}_{2} \mathrm{O}\right)_{4}$ \\
\hline $3 \leftarrow 2$ & 0.0 & 0.0 \\
$4 \longleftarrow 3$ & 1.0 & 2.4 \\
$5 \leftarrow 4$ & 3.0 & 4.4 \\
$6 \longleftarrow 5$ & 5.4 & 7.6 \\
$7 \leftarrow 6$ & 8.2 & 12.0 \\
$8 \leftarrow 7$ & 12.0 & 18.1 \\
$9 \longleftarrow 8$ & 16.8 & \\
$4 \leftarrow 5$ & 2.9 & \\
$5 \leftarrow 6$ & 5.4 & \\
$6 \leftarrow 7$ & 7.0 & \\
$7 \leftarrow 8$ & 11.8 &
\end{tabular}

${ }^{a}$ Both bands are approximately linear as a function of $J(J+1)$ in either the ground or excited vibrational states. See Figures 7 and 8.

$\left(\mathrm{D}_{2} \mathrm{O}\right)_{4}$ data set, wherein the averaged line positions of each doublet (the doublet centers) were fit, and $\alpha$ was determined by averaging the spacing of each doublet. The results of the fit of eq 2 to the $\left(\mathrm{H}_{2} \mathrm{O}\right)_{4}$ data are shown in Table 2. The $\left(\mathrm{D}_{2} \mathrm{O}\right)_{4}$ parameters are included for comparison. Although the doublet splittings are likely to be present in both upper and lower vibrational states, the fit assumed that they were only present in the upper state for reasons of convenience. More precisely, $\alpha$ is either the sum or difference of the actual energy level splittings present in both vibrational states.

It is difficult to determine whether an intensity difference is present in any comparison of the $2.26 \mathrm{GHz}$ doublet components. Although relative intensities of lines within a few hundred $\mathrm{MHz}$ are reasonably reliable, the output power of the far-IR laser varies too much as a function of frequency to allow for reliable intensity relations to be made between lines separated by more than that amount. The fact that there are missing lines in the lower frequency subband (Table 1 and Figure 3) is more a function of chance combinations of low laser sideband power, strong atmospheric water absorptions and/or laser coverage gaps in those regions, than of intrinsically weaker signals. Many cases where the doublet intensities were approximately equal were observed. In the end, we conclude that the doublets are of nearly equal intensity, conservatively reliable to within $\pm 15 \%$.

In our second $\left(\mathrm{D}_{2} \mathrm{O}\right)_{4}$ study, ${ }^{46}$ additional splittings in the $K$ $=2$ manifold of transitions were reported. Similar splittings, increasing nonlinearly with $J$, were found in the $K=2$ transitions of the $\left(\mathrm{H}_{2} \mathrm{O}\right)_{4}$ data. They are listed in Table 3 and discussed further in section VI. Like their counterparts in the $\left(\mathrm{D}_{2} \mathrm{O}\right)_{4}$ spectrum, the $K=2$ transitions (doubled doublets) are split approximately as $J^{2}$ [or $\left.J(J+1)\right]$, and appear to be present in both upper and lower states of the spectrum. This is realized 


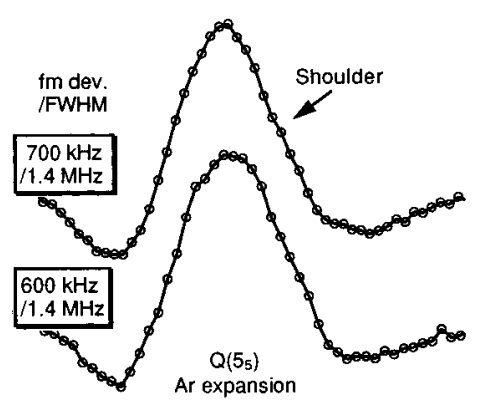

Figure 5. Signal averaged, undermodulated scans of the $5_{5} \leftarrow 5_{5}$ transition of the $67.9 \mathrm{~cm}^{-1}\left(\mathrm{H}_{2} \mathrm{O}\right)_{4}$ band. The $1.4 \mathrm{MHz}$ line widths are approximately 1.5 times broader than $\left(\mathrm{H}_{2} \mathrm{O}\right)_{3}$ transitions found in the same region. The line broadening, in addition to the weak shoulder apparent on the high-frequency side of the peaks is indicative of unresolved fine structure in the lines. Other transitions in the spectrum gave similar results.

by recognizing that the splittings are different for the $\mathrm{P}$ and $\mathrm{R}$ branch lines with common upper or lower state $J$, although in the case of the $\left(\mathrm{H}_{2} \mathrm{O}\right)_{4}$ spectrum, only one P-branch splitting was measured due to the weakness of the band, which is exacerbated in the $K=2$ case by the extra splitting. No such splittings were observed in either of the Q-branches. The highest $J^{\prime \prime}{ }_{K=2} \rightarrow J^{\prime}{ }_{K=2}$ Q-branch transition observed had $J^{\prime \prime}=J^{\prime}$ $=5$. The fact that splittings were observed in $\mathrm{R}\left(5_{2}\right)$ but not in $\mathrm{Q}\left(5_{2}\right)$ indicates that the energy levels are similarly split in the upper and lower states, and that the transitions likely represent the difference between the two manifolds, consistent with the conclusion in the previous study. ${ }^{46}$

As illustrated in Figure 4, each transition in the spectrum was broadened by roughly 1.5 times the normal Doppler limited line width (fwhm) of $\sim 1 \mathrm{MHz}$ at $70 \mathrm{~cm}^{-1}$. Indeed, a $\left(\mathrm{H}_{2} \mathrm{O}\right)_{3}$ spectrum was recorded in the same spectral region and exhibited the expected line widths, as shown. Attempts to resolve any underlying structure revealed at least one shoulder on the blue side of each peak (Figure 5). No additional resolution was achieved in scans using $\mathrm{Kr}$ as the carrier gas which, in principle, should decrease the intrinsic line widths by $2^{1 / 2}$ by lowering the average speed of the supersonic expansion.

Finally, Table 1 shows that a small coriolis-type perturbation is operative in the high $J$ transitions observed in the $J=K$ portion of each of the Q-branches, although it is more pronounced in the lower frequency tunneling doublet component. This deviation can be accounted for by fitting higher order centrifugal distortion terms to the data, but may also reflect a very slight $J$ dependence of the principal $2.26 \mathrm{GHz}$ tunneling splitting. Only quartic centrifugal distortion parameters were used in the fit that produced the residuals listed in Table 1.

\section{Group Theoretical Analysis}

The value of permutation-inversion (PI) group theory ${ }^{64}$ in rationalizing rearrangement dynamics of water and other nonrigid clusters has been well established. Dyke's treatment ${ }^{65}$ of the nonrigid water dimer rearrangements has served as a prototype for understanding the dynamics of larger water clusters. In the prototypical-if complicated-cases of the water and ammonia dimers, all possible rearrangement dynamics give rise to spectral effects and therefore serve to confirm the group theoretical analyses. The water trimer presented a case wherein one possible structural rearrangement, the so-called cw $\leftrightarrow \mathrm{ccw}$ tunneling-an analogue to donor-acceptor interchange tunneling in the water dimer-was not observed in the VRT spectra, allowing for a simplification of the PI treatment, but with a concomitant loss of information about the IPS. ${ }^{8}$ The influences of nonadditive intermolecular forces and more favorable $\mathrm{H}$ -
TABLE 4: Rovibrational Selection Rules for the Water Tetramer under the PI Group $C_{4 h}(\mathrm{M})^{a}$

\begin{tabular}{clll}
\hline \multirow{2}{*}{$\begin{array}{c}\text { vibrational transition } \\
\Gamma_{\text {vib }}^{\prime \prime} \rightarrow \Gamma_{\text {vib }}^{\prime}\end{array}$} & \multicolumn{1}{c}{$\begin{array}{c}\text { rotational } \\
\text { selection rules }\end{array}$} & \multicolumn{2}{c}{ prediction $/ \mathrm{cm}^{-1}$} \\
\hline $\left.\mathrm{H}_{2} \mathrm{O}\right)_{4}$ & $\left(\mathrm{D}_{2} \mathrm{O}\right)_{4}$ \\
\hline $\mathrm{B}_{\mathrm{g}}$ & $\Delta K=0$ & 79,261 & 77,247 \\
$\rightarrow \mathrm{E}_{1 \mathrm{~g}}$ & $\Delta K= \pm 1$ & 236 & 175 \\
$\rightarrow \mathrm{E}_{2 \mathrm{~g}}$ & $\Delta K= \pm 1$ & 236 & $175^{\circ}$ \\
$\rightarrow \mathrm{A}_{\mathrm{u}}$ & $\Delta K=0$ & 199 & 142 \\
$\rightarrow \mathrm{E}_{1 \mathrm{u}}$ & $\Delta K= \pm 1$ & 254 & 235 \\
$\rightarrow \mathrm{E}_{2 \mathrm{u}}$ & $\Delta K= \pm 1$ & 254 & 235
\end{tabular}

${ }^{a}$ Predictions of the eight lowest intermolecular vibrational frequencies of Schütz et al. ${ }^{38}$ are also given. ${ }^{b}$ Predictions from the ab initio calculation of Schütz et al. ${ }^{38}$

bonding geometries present in larger complexes appear to effectively quench some of the otherwise observable dynamics by increasing the heights of the associated rearrangement barriers. Indeed, no tunneling splittings of any kind were observed in two VRT bands of $\left(\mathrm{D}_{2} \mathrm{O}\right)_{5}$, although it is worth noting that analysis of an $\left(\mathrm{H}_{2} \mathrm{O}\right)_{5}$ spectrum which appears to contain resolved splitting patterns is presently underway. ${ }^{66}$

A PI group theoretical treatment was applied to the tunneling splittings in the $\left(\mathrm{D}_{2} \mathrm{O}\right)_{4}$ spectrum in order to rationalize the fine structure. ${ }^{46}$ Only a brief summary of that work will be given here. Generally, in such group theoretical analyses, the smallest subgroup of the complete nuclear permutation-inversion (CNPI) group $^{64}$ which rationalizes all of the observable or "feasible" dynamics - those that can be observed in the experiments of interest-is sought. The CNPI group, the size of which is given in general by $2(a$ !, b!, ..., z!) for a nonrigid system containing (a) copies of atom A, (b) copies of B, and so forth, grows exponentially with cluster size to unmanagable proportions (the CNPI group of the water tetramer contains 1935360 elements), but can usually be pared down considerably by recognizing that chemical $\mathrm{OH}$ bond breaking is unobservable in the VRT experiments. The largest group of feasible tetramer operations can be expressed by the Wreath product ${ }^{67} S_{4}\left[S_{2}\right]$, where $S_{4}$ and $S_{2}$ are the groups of all permutations of four and two identical objects, respectively. This group can also be expressed by the direct product $S_{4} \otimes\left[S_{2}\right]^{4} \otimes\left\langle\mathrm{E}, \mathrm{E}^{*}\right\rangle$, which contains 768 symmetry operations that interconvert between uniquely labeled frameworks representing the same equilibrium structure $\left(E^{*}\right.$ is the operation that inverts all nuclear and electronic coordinates through the center of mass).

In the case of the tetramer, it was more convenient to use a "building-up" procedure to determine the MS group. Because the theoretical consensus is that the equilibrium structure has $S_{4}$ point group symmetry, the PI group $S_{4}(\mathrm{M})$ is the smallest possible tetramer MS group. The symmetry operations of $S_{4}(\mathrm{M})$ and their effects on the coordinates were given in ref 46. $S_{4}(\mathrm{M})$ is isomorphic to the point group of a rigid $S_{4}$ tetramer, therefore it does not rationalize any of the observed splittings, and a larger group is required. In the previous studies, a concerted flipping operation (see Figure 1), wherein all of the unbound protons (deuterons) simultaneously flip from one side of the $\mathrm{O}-\mathrm{O}-$ $\mathrm{O}-\mathrm{O}$ plane to the other, was proposed to explain the measured doublets. Since that work, the theoretical study of Gregory and Clary ${ }^{58}$ and the new $\left(\mathrm{H}_{2} \mathrm{O}\right)_{4}$ data have combined to strengthen that hypothesis. Together with the operations of $S_{4}(\mathrm{M})$, the PI operations that generate the concerted flipping motion form a group of eight PI operations called $C_{4 h}(\mathrm{M})$. The character table and the symmetry labeling of the VRT states under $C_{4 h}(\mathrm{M})$ were derived in ref 46 . The possible intermolecular vibrational transitions allowed by the $C_{4 h}(\mathrm{M})$ selection rules are listed in Table 4. The overall $C_{4 h}(\mathrm{M})$ selection rules are $\mathrm{A}_{\mathrm{g}} \leftrightarrow \mathrm{A}_{\mathrm{u}}, \mathrm{B}_{\mathrm{g}}$ $\leftrightarrow \mathrm{B}_{\mathrm{u}}, \mathrm{E}_{1 \mathrm{~g}} \leftrightarrow \mathrm{E}_{2 \mathrm{u}}$, and $\mathrm{E}_{2 \mathrm{~g}} \leftrightarrow \mathrm{E}_{1 \mathrm{u}}$. 
The bifurcation tunneling operations $12,34,56$, and 78 , and their products, e.g., (12)(34), (12)(34)(56), and (12)(34)(56)(78) form a group of 14 elements. The direct product of this group with $C_{4 h}(\mathrm{M})$ leads to a group of 120 elements, called $\mathrm{G}_{120}$, which describes a tetramer undergoing flipping inversion and bifurcation tunneling. The operation (12) exchanges the coordinates of the bound and free protons or deuterons of monomer A, operation (34) of monomer B, and so on. In general, for cyclic water clusters such as the trimer, tetramer, and pentamer, similar group theoretical treatments predict splitting of each rotation-vibration state into as many as $n+$ 1 separate levels, where $n$ is the number of monomers. Fourcomponent splitting patterns are observed in both $\left(\mathrm{H}_{2} \mathrm{O}\right)_{3}$ and $\left(\mathrm{D}_{2} \mathrm{O}\right)_{3}$ VRT spectra, where two of the possible six components are degenerate and not usually resolved. ${ }^{6,68}$ Therefore each component of the doublets in the $\left(\mathrm{H}_{2} \mathrm{O}\right)_{4}$ spectrum could be a convolution of as many as five individual lines comprising fine structure due to bifurcation tunneling.

\section{Tunneling Splittings}

In a series of calculations, Gregory and Clary $20,53,58$ have applied the DQMC simulation technique toward calculation of the vibrationally averaged structural parameters and tunneling splittings of the water dimer through pentamer. In a recent study of the tetramer, ${ }^{58}$ they used two potentials, ASP-NB, a manybody forces optimized version of Millot and Stone' $\mathrm{s}^{69}$ water dimer IPS, and NEMO, an empirical potential developed by Astrand and co-workers. ${ }^{70}$ The latter contained a many-bodyiterated induction term ${ }^{71}$ and the Axilrod-Teller-triple dipole dispersion term ${ }^{72}$ to model many body interactions. While the ASP-NB potential failed to produce tunneling splittings in the simultaneous flipping coordinate, the NEMO potential predicted ground-state tunneling splittings of 17 and $1200 \mathrm{MHz}$ for $\left(\mathrm{D}_{2} \mathrm{O}\right)_{4}$ and $\left(\mathrm{H}_{2} \mathrm{O}\right)_{4}$, respectively. The VRT data are transitions between levels and as such, can usually only measure sums or differences of split levels, depending on the selection rules. Although it is conceivable that upper and lower state splittings which are large could have a small difference, thus producing small spectral splittings, it is more likely that the spectral splittings will scale roughly with the size of the energy level splittings themselves. The splittings predicted by Gregory and Clary ${ }^{58}$ are of the correct order of magnitude to rationalize the measured line spacings of 5.6 and $2260 \mathrm{MHz}$.

Two alternative explanations for the origin of the $\left(D_{2} \mathrm{O}\right)_{4}$ doublet splittings outlined in ref 46 are a nondegenerate tunneling scheme and one in which the tunneling splittings occur only in the upper vibrational state. In the latter, the tunneling motion, not feasible in the lower vibrational state, is facilitated by vibrational excitation in the tunneling coordinate in the upper level, effectively lowering the barrier. We are now in a better position to rule out the possibility of the nondegenerate tunneling mechanism suggested by Wales (refer to ref 46 and to Wales ${ }^{73}$ for further discussion of nondegenerate tunneling). That possibility seems doubtful because IPS minima supporting non degenerate structural frameworks would be unlikely to support identically spaced rotational manifolds, as have been observed (for $J \leq 15$ ) in both of the tetramer spectra measured to date. The possibility of a vibrationally facilitated tunneling motion rested upon the argument that the vibrational excitation contained significant motion in the flipping coordinates. If the vibrational assignment to be proposed in the next section is correct, then this possibility seems less likely because little $\mathrm{H}$-bond torsional excitation would be expected to be present in the assigned mode.

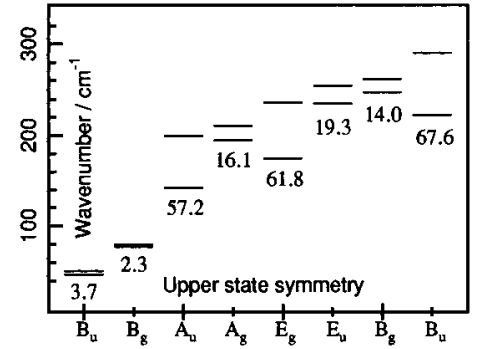

Figure 6. Isotope shifts of the lowest eight water tetramer normal modes, as calculated by Schütz et al. ${ }^{38}$ The $\mathrm{D}_{2} \mathrm{O}$ frequency is shown by the lower line and the symmetry label is that of the upper vibrational level. Small, intermediate, and large shifts are present, indicative of the degree of participation (or cancellation) of the flipping coordinates in the mode.

No evidence of bifurcation tunneling splittings was observed in the $\left(\mathrm{D}_{2} \mathrm{O}\right)_{4}$ data. Each line in the spectrum exhibited normal Doppler broadened line widths of approximately $1 \mathrm{MHz}$. In contrast, the line broadening observed in the $\left(\mathrm{H}_{2} \mathrm{O}\right)_{4}$ data (Figure 4) is strongly indicative of unresolved bifurcation tunneling splittings. Figure 5 shows two undermodulated scans of the $\mathrm{Q}\left(5_{5}\right)$ transition. A weak shoulder, the only partially resolved structure, is evident on the blue side of the peak when scanned at frequency modulation deviations below $1 \mathrm{MHz}$. There is no doubt that each line in the spectrum is broadened by unresolved substructure.

\section{Vibrational Analysis}

In ref 46, we tentatively proposed that the most likely candidate for a vibrational assignment of the $\left(\mathrm{D}_{2} \mathrm{O}\right)_{4}$ spectrum was the $A_{g} \rightarrow A_{u}$ transition, predicted by Schütz and coworkers $^{38}$ to be near $142 \mathrm{~cm}^{-1}$. This assignment was based in part on symmetry considerations (see Table 4) and in part upon the assumption that ab initio calculated harmonic frequencies would be too high by up to a factor of 2 , an assumption not inconsistent with many other results on weakly bound systems. The new $\left(\mathrm{H}_{2} \mathrm{O}\right)_{4}$ spectrum has provided cause to reevaluate that assignment. The close proximity of the band origins of the tetramer- $d_{8}$ and $-h_{8}$ spectra (they are separated by only 0.09 $\mathrm{cm}^{-1}$ ) can only be rationalized in one of two ways: (1) the two transitions are not due to excitations in the same vibrational coordinate, and the proximity of the bands is a coincidence, or (2) they are due to an identical vibration which is not significantly shifted by complete $\mathrm{H} \leftrightarrow \mathrm{D}$ substitution. The lowest eight normal-mode frequencies of both pure isotopomers of the tetramer from the calculation of Schütz et al., ${ }^{38}$ are shown graphically in Figure 6. The $\left(\mathrm{H}_{2} \mathrm{O}\right)_{4}$ frequencies are essentially the same as those calculated by Xantheas and Dunning. ${ }^{12}$ From the figure, it is clear that there are modes for which $\mathrm{H} \leftrightarrow \mathrm{D}$ substitution produces large shifts and those wherein the shift may be insignificant to within the precision of the calculation. In particular, the two lowest frequency vibrations predicted at $50.5[46.8]$ and $79.4[77.1] \mathrm{cm}^{-1}$, for $\left(\mathrm{H}_{2} \mathrm{O}\right)_{4}\left[\left(\mathrm{D}_{2} \mathrm{O}\right)_{4}\right]$, have isotope shifts of only $7 \%$ and $3 \%$, respectively, after substitution of $\mathrm{D}$ for $\mathrm{H}$ in all positions. The reason for the small shifts can be seen in the normal coordinate diagrams of Schütz et al. ${ }^{38}$ They show that in those modes, (quasi) four-membered planar ring deformation and ring bending vibrations, the normal coordinate vectors of the atoms in each monomer all lie in essentially the same direction, i.e., there is no significant rotation of the monomers involved in the motion. Insignificant monomer rotations coupled with these vibrations could yield fortuitous cancellation of part of the already small calculated differences. In Xantheas' MP2 calculation, ${ }^{12}$ the intensity of the 50.5 [46.8] $\mathrm{cm}^{-1}$ transition is less than $0.001 \mathrm{~km} /$ mole. 


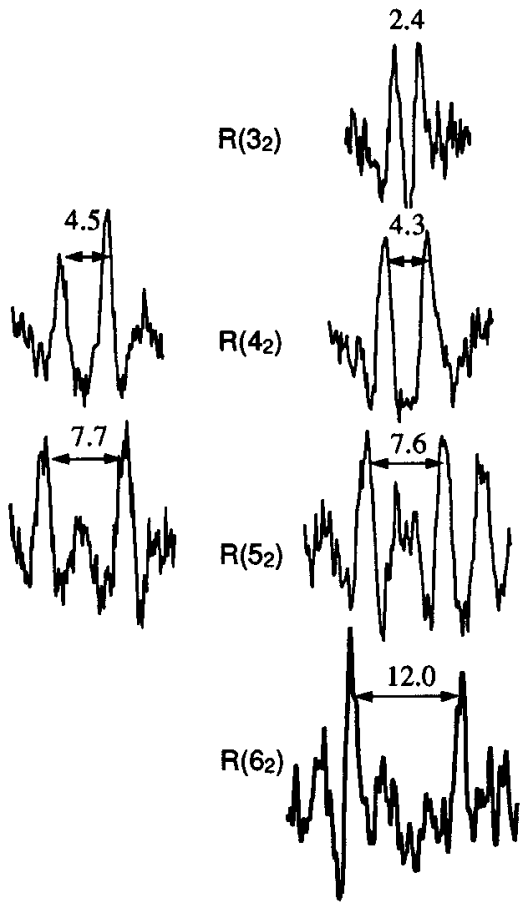

Figure 7. The resolved $K=2 \mathrm{R}$-branch splittings. The $\mathrm{R}\left(3_{2}\right)$ and $\mathrm{R}\left(6_{2}\right)$ lower components of the principal tunneling doublet were not found.

From Table 4, we see that the transition $\mathrm{A}_{\mathrm{g}} \rightarrow \mathrm{B}_{\mathrm{g}}$ would be expected to yield a $\Delta K=0$ spectrum. Given the proximity of the two bands, the similarity of the splittings in the $K=2$ transitions, and the compatibility of the isotope shifts and selection rules with the predicted vibrations, it seems reasonable to reassign both spectra to be due to the lowest $A_{g} \rightarrow B_{g}$ transition predicted to occur at $77-79 \mathrm{~cm}^{-1}$. It is surprising that the experimental results would be so close to the harmonic vibrational frequencies. Such calculations are typically too high by as much as a factor of 2 due to the large anharmonicities associated with the weak H-bonds. Although it is likely that the mode in question contains significant coupling to other intermolecular coordinates, the closeness of the experimental and theoretical results may reflect a more rigid tetramer framework arising from increased per-monomer binding energies and the strongly concerted motion required by the high symmetry of the cluster.

\section{VI. $K=2$ Splittings}

As observed previously in the $\left(\mathrm{D}_{2} \mathrm{O}\right)_{4}$ spectrum, each component of the $K=2$ transition doublets $J_{2}{ }^{\prime} \leftarrow J_{2}{ }^{\prime \prime}$ is further split into a doublet in the $\left(\mathrm{H}_{2} \mathrm{O}\right)_{4}$ spectrum. Some of the R-branch splittings are shown in Figure 7. Due to the relative weakness of the P-branch and the fact that each $K=2$ doublet is half as intense as an unsplit $K=2$ transition would be, only one P-branch doublet was measured. However, that measurement shows that the $K=2$ splittings behave in a manner similar to those in the $\left(\mathrm{D}_{2} \mathrm{O}\right)_{4}$ spectrum, namely that they differ as a function of either upper or lower state $J$ in the $\mathrm{P}$ and $\mathrm{R}$ branches. The difference shows that the splitting is present in $K=2$ rotation-tunneling levels of both the upper and lower vibrational states. The $\mathrm{R}$ branch $K=2$ splittings are plotted as a function of lower state $J(J+1)$ in Figure 8. The $J(J+1)$ dependence of the splittings, evident in the figure, is indicative of a coriolistype interaction. Similar splitting patterns have been analyzed in the $\left(\mathrm{H}_{2} \mathrm{O}\right)_{3}$ spectrum observed by Liu and co-workers ${ }^{74}$ near $87.1 \mathrm{~cm}^{-1}$. They were rationalized in terms of kinetic coupling of internal rotational motions (flipping tunneling in that case) with the overall rotation.

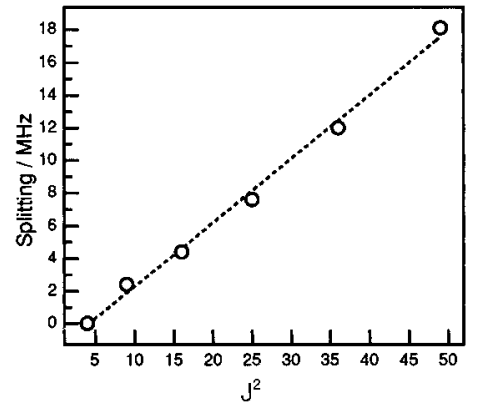

Figure 8. A plot of the $K=2$ R-branch splittings vs $J(J+1)$ shows that the splittings behave as expected for a coriolis splitting in either or both of the ground and upper vibrational levels.

\section{Vibrationally Averaged Structure}

With the goal of extracting a reliable estimate of $R_{\mathrm{OO}}$ from the new data, a structural analysis identical with the one described in refs 45 and 46 was undertaken. Because the tetramer is a symmetric top in both the VRT data and the equilibrium structure calculations, the complexity of a structural determination of a system with 18 intermolecular degrees of structural freedom is reduced considerably. The symmetry of the system, whether from dynamic averaging or rigorous symmetry of the global minimum, allows a structure to be determined by determining the coordinates of just one monomer. Rigorously, six coordinates are needed to fix the structure exactly, three to fix the translation of the given monomer in the cluster, and three to specify its intramolecular coordinates - two $\mathrm{O}-\mathrm{H}$ bond lengths and the $\mathrm{HOH}$ angle. The VRT data at hand contain only one piece of structural information, viz., the B rotational constant, which is inversely proportional to the (equal) moments of inertia about axes perpendicular to the oblate top $c$-axis, and is therefore a function of $R_{\mathrm{OO}}$. By fixing five of the coordinates to calculated values, $R_{\mathrm{OO}}$ can be estimated. Using the ab initio structural parameters of Xantheas and Dunning, ${ }^{12}$ which contain distorted (relaxed) monomers, an estimate of $R_{\mathrm{OO}}$ $=2.79 \AA$ was obtained by varying the distance between monomers until the calculated and observed $\mathrm{B}$ constants were the same. The $R_{\mathrm{OO}}$ estimate is in exact agreement with that determined from the $\left(\mathrm{D}_{2} \mathrm{O}\right)_{4}$ data. ${ }^{45,46}$

Observation of a $\Delta K= \pm 1$ spectrum would allow for a more rigorous determination of the structure because, in addition to providing a second data point, it would allow measurement of the moment of inertia $\left(I_{\mathrm{c}}\right)$ about the oblate rotor symmetry axis. That property is more sensitive to the heavy atom (oxygen) positions than the $I_{\mathrm{a}}=I_{\mathrm{b}}$ moments. Liu and co-workers ${ }^{7}$ have modeled the vibrationally averaged structure of the water trimer using a Monte Carlo integration scheme to integrate over the torsional-spacial wave functions of Gregory and Clary ${ }^{20}$ and thereby calculate vibrationally averaged rotational constants and structural parameters. This is a somewhat more realistic treatment of the structural averaging in that it reflects the quantum mechanically most probable positions of the free protons or deuterons. A similar treatment of the tetramer, perhaps constraining opposite pairs of free protons or deuterons to move together, might be insightful. However, given the level of approximation already involved, the present calculation is probably sufficient until another structural parameter can be determined experimentally. Furthermore, the tetramer $R_{\mathrm{OO}}$ fits quite well with the exponentially decreasing trend predicted by Xantheas ${ }^{18}$ and others as a function of increasing cyclic cluster size, as was shown in refs 45,46 , and 1 . 
TABLE 5: Comparison of Several Low-Energy Torsional Variants of the Water Tetramer from the Calculation of Schiitz $^{a}$

\begin{tabular}{cc}
\hline structure & $\Delta E_{\text {rel }}$ \\
\hline (uudd) & 0.93 \\
(uupd) & 1.24 \\
(uudp) & 1.24 \\
(uppd) & 1.38 \\
(updp) & 1.40 \\
(uuuu) & 2.18 \\
(pppp) & 2.79
\end{tabular}

${ }^{a} \Delta E_{\text {rel }}(\mathrm{kcal} / \mathrm{mol})$ is the energy relative to the global minimum (udud) structure. In this notation "u" and "d" stand for free $\mathrm{H}$ or D atoms above and below the $\mathrm{O}-\mathrm{O}-\mathrm{O}-\mathrm{O}$ plane (or near plane), respectively, and "p" indicates H or D in the plane. All of these structures are saddle points except (uudd).

\section{Discussion}

$\left(\mathrm{H}_{2} \mathrm{O}\right)_{4}$ and $\left(\mathrm{D}_{2} \mathrm{O}\right)_{4}$ VRT spectra have now qualitatively confirmed that the equilibrium structure of the water tetramer is one of the cyclic, sequentially $\mathrm{H}$-bonded torsional variants predicted by theory to be the equilibrium structure. Although the moments of inertia about the oblate top $(c)$ inertial axis cannot be directly determined from either data set, the fact that Q-branch lines for $J>15$ (Figure 3) are observed, and the shape of the Q-branch intensity profile itself, are strongly indicative of an oblate rotor spectrum. The intensities would drop off much faster as a function of $K$ for a less oblate cluster. Observation of a $\Delta K= \pm 1$ spectrum would greatly facilitate a more rigorous determination of the vibrationally averaged structure. The results of the vibrational and group theoretical analysis (Table 4$)$ predict that such a $\left(\mathrm{D}_{2} \mathrm{O}\right)_{4}$ spectrum may be found near $175 \mathrm{~cm}^{-1}$, and its $\left(\mathrm{H}_{2} \mathrm{O}\right)_{4}$ counterpart near $236 \mathrm{~cm}^{-1}$. Assuming that the harmonic prediction overestimates the transition frequency, the $\left(\mathrm{D}_{2} \mathrm{O}\right)_{4}$ transition may lie near the strong, well-known $70 \mu \mathrm{m}\left(143 \mathrm{~cm}^{-1}\right)$ far-IR laser. Fellers and co-workers ${ }^{75}$ have also recorded a $\Delta K= \pm 1$ spectrum which is apparently due to $\left(\mathrm{D}_{2} \mathrm{O}\right)_{4}$ near $510 \mathrm{~cm}^{-1}$, but it has thus far eluded a definitive analysis.

Observation of a planar oblate tetramer, as noted previously, ${ }^{45,46}$ is consistent with the electric field gradient deflection experiments of Dyke and Muenter, ${ }^{76}$ who bracketed the dipole moments of clusters larger than the dimer to be much smaller than the monomer moment of 1.4 D. The dipole moment of the $S_{4}$ tetramer structure is zero by symmetry. Both the observations of Dyke and Muenter and our VRT experiments are consistent with the results of Haberland and co-workers, ${ }^{77-79}$ who were unable to attach low energy electrons to dipole bound states of the tetramer, but observed mass signals for $\left(\mathrm{H}_{2} \mathrm{O}\right)_{n}{ }^{-}$, for $n=2,3,5, \ldots$ Those experiments limit the tetramer dipole moment to lie below that of the classical threshold ${ }^{80}$ for attaching dipole-bound electrons, $\mu_{\min }=1.8 \mathrm{D}$.

The consensus of most theoretical studies is that the $S_{4}$ structure is the lowest energy configuration, but Schütz et al. ${ }^{38}$ have shown that several cyclic variants, differing mainly in the $\mathrm{H}$-bond torsional coordinates, have stabilization energies very close to the minimum. Some of their results and notation definitions are summarized in Table 5. The energy difference between the (udud) and (pppp) structures is $2.79 \mathrm{kcal} \mathrm{mole}^{-1}$. Xantheas ${ }^{12}$ estimated the zero-point energy using the (udud) configuration to be $8.28 \mathrm{kcal} \mathrm{mole}^{-1}$, or roughly $1 / 3$ of the stabilization energy.

In their recent DQMC study, Gregory and Clary ${ }^{58}$ incorporated the effect of the zero point energy in a calculation of the simultaneous flipping and calculated the bifurcation tunneling splittings in the water tetramer and pentamer. Using two model
IPSs, the ASP-NB potential of Millot and Stone, ${ }^{69}$ and the additive NEMO potential of Astrand and co-workers, ${ }^{70}$ they calculated flipping inversion splittings of 17 and $1200 \mathrm{MHz}$ for $\left(\mathrm{D}_{2} \mathrm{O}\right)_{4}$ and $\left(\mathrm{H}_{2} \mathrm{O}\right)_{4}$, respectively, but only with the latter IPS. They noted that in their simulations, the ASP-NB surface leads to probability density on only one side of the $\mathrm{O}-\mathrm{O}-$ $\mathrm{O}-\mathrm{O}$ framework, i.e., it does not allow flipping. Incidentally, in the same study, Gregory and Clary surmised that flipping must occur because the VRT spectra are due to a symmetric top. In Section I it was noted that this is not necessarily the case. Because the $S_{4}$ equilibrium structure is a symmetric top, no a priori evidence of flipping is obtained from the spectrum, except for the tunneling splittings.

While the flipping tunneling scheme has been reinforced by the $\left(\mathrm{H}_{2} \mathrm{O}\right)_{4}$ data and by the calculation of Gregory and Clary, the nondegenerate tunneling scenario proposed by Wales and described in ref 46 seems a less likely candidate for the source of the splittings. Nondegenerate rearrangements may indeed be occurring in the cyclic tetramer, but they are not likely to be the source of the doublet splittings observed in the data because such splittings would not be expected to be $J$-independent.

The VRT data cannot yet conclusively rule out the possibility that the tunneling splittings are only present in the upper vibrational state and are facilitated by vibrational excitation in the flipping inversion coordinate. However, if the vibrational analysis in Section V is accurate, such excitation is probably minimal. Considering the data and symmetry arguments, the most likely scenario is that flipping inversion is active in both the lower and upper vibrational states and that the observed splittings are either a sum or difference, depending on the ordering of the tunneling doublet symmetry species, of the split rotational states therein.

No evidence of bifurcation tunneling splittings was observed in the $\left(\mathrm{D}_{2} \mathrm{O}\right)_{4}$ spectrum, and no such splittings were actually resolved in the $\left(\mathrm{H}_{2} \mathrm{O}\right)_{4}$ study, although their presence is strongly indicated by broadening of all of the VRT transitions and observation of possible substructure in the lines. Gregory and Clary calculated bifurcation tunneling splittings of 220 and 70 $\mathrm{MHz}$ for $\left(\mathrm{H}_{2} \mathrm{O}\right)_{5}$ and $\left(\mathrm{D}_{2} \mathrm{O}\right)_{5}$, respectively, but did not perform analogous calculations for the tetramer. These splittings are smaller by roughly a factor of 2 than the corresponding splittings they calculated for the trimer. ${ }^{53}$ No bifurcation tunneling splittings were observed in either of two published $\left(\mathrm{D}_{2} \mathrm{O}\right)_{5}$ VRT spectra. ${ }^{47,48}$ Brown, ${ }^{66}$ however, is presently analyzing a new $\left(\mathrm{H}_{2} \mathrm{O}\right)_{5}$ spectrum which appears to exhibit resolved splittings indicative of bifurcation tunneling. If the VRT splittings represent the difference between the energy level splittings in the lower and upper vibrational states and the splittings are identical in both, no spectral doubling will be observed. If the transitions measure the sum, a spectral splitting will result. Although not required, it is not unreasonable to expect the spectral manifestation in either case to be of the same order of magnitude as the energy level splittings themselves. Insofar as that holds, the flipping inversion observations are consistent with the results of Gregory and Clary, as are the pentamer results. The observed spectral bifurcation splittings of 0 and $<1.5 \mathrm{MHz}$ for $\left(\mathrm{D}_{2} \mathrm{O}\right)_{4}$ and $\left(\mathrm{H}_{2} \mathrm{O}\right)_{4}$, respectively, are of the same order as the predicted splittings in the pentamer, which are likely to be similar to those in the tetramer.

Because the (uudd) structure lies within $5 \%$ of the total stabilization energy of the global minimum, torsional motions interconverting (udud) and (uudd) structures may occur on time scales on the order of flipping in the trimer, which has been shown to be fast. Schütz and co-workers enumerated a pseudorotation pathway for this structure, (uudd) $\rightarrow$ (uddu) $\rightarrow$ 
TABLE 6: Comparson of the ab Initio Tetramer Energetics (kcal/mol) of Koehler and Co-Workers ${ }^{40}$ and of Xantheas ${ }^{18 a}$

\begin{tabular}{lccccc}
\hline & \multicolumn{2}{c}{ Koehler et al. } & & \multicolumn{2}{c}{ Xantheas } \\
\cline { 2 - 3 } \cline { 5 - 6 } energy & MP3/6-31G** & \% cont & & MP4/aug-cc-pVDZ & \% cont \\
\hline$\Delta E(2)$ & -18.41 & 71.0 & & -14.80 & 62.2 \\
$\Delta E(2)_{\text {nnn }}$ & -2.96 & 11.4 & & -3.18 & 13.4 \\
$\Delta E(3)$ & -4.70 & 18.1 & & -6.16 & 25.9 \\
$\Delta E(4)$ & -0.27 & 1.0 & & -0.55 & 2.3 \\
$\Delta E_{\text {relaxation }}$ & 0.41 & -1.6 & & 0.8 & -3.7 \\
$\Delta E_{\text {total }}$ & -25.93 & & & -23.80 &
\end{tabular}

${ }^{a}$ Fractional contribution of each of the terms to the total stabilization energy (\% cont.) is also shown. $\Delta E(n)$ is the n-body contribution, $\Delta E(2)_{\mathrm{nnn}}$ is the pairwise additive contribution from nonnearest neighbor interactions, and $\Delta E_{\text {relaxation }}$ is the contribution from distortion of the monomers.

(dduu) $\rightarrow$ (duud) $\rightarrow$ (uudd), analogous to the pathway that led to pseudorotation treatments of torsional vibrations in the trimer. ${ }^{38}$ If the (udud) $\leftrightarrow$ (uudd) rearrangement is active, then low-frequency manifold of pseudorotation states might also be present in the tetramer, making the density of low-frequency levels much higher than the harmonic calculations might indicate. It is also possible that the addtional $K=2$ doublings are a manifestation of sampling of these additional minima. Continued far-IR searches, which could measure a different quasi-planar structure, will best answer this question.

Several theoretical studies have examined the contribution of many-body forces in the tetramer, ${ }^{18,31,37,40,81,82}$ usually as part of a systematic study of nonadditivity as a function of cluster size. These have shown that the four-body term in the potential expansion contributes on the order of $1 \%$ to the stabilization energies, and probably can be ignored for purposes of cluster structure prediction and liquid and solid water simulations. The three-body contribution to stabilization of the tetramer may be quite large, up to $30 \%$ of the total. Results of the high-level (MP2) calculations of Koehler et al. ${ }^{40}$ and of Xantheas ${ }^{18}$ are reproduced in Table 6 . They show that, while $10 \%$ of the stability of the tetramer arises from nonnearest neighbor interactions, up to $25 \%$ is due to three-body forces. Clementi and co-workers, ${ }^{15}$ and Chałasiński et al., ${ }^{22}$ among others, have shown that the principal many-body contribution in cyclic water clusters arises from many-body induction which is reinforced in cyclicly $\mathrm{H}$-bonded structures. Each monomer dipole moment induces a polarization in the neighboring monomer, thus enhancing its dipole moment and ability to polarize the next water in the sequence, and so on. It is principally this interaction that leads to the dipole moment enhancement of monomers comprising the bulk phases. ${ }^{82}$ The dipole moment of the water hexamer was recently measured by Liu and co-workers, ${ }^{83}$ providing direct insight into this polarization enhancement. In a detailed study of nonadditivity in the trimer, Chałasiński et al. ${ }^{22}$ also showed that the additive intermolecular forces, as well as the three-body induction, dispersion and exchange forces in water are quite anisotropic, with the latter contributing in the direction of straightening the $\mathrm{H}$-bonding angles, $\delta(\mathrm{O}-\mathrm{H} \cdots \cdot \mathrm{O})$. A study similar to their water trimer work would be helpful in understanding stabilization of the tetramer and larger clusters wherein the H-bonds angles are closer to linear and the contribution of three body forces is more pronounced.

The tetramer inter-oxygen separation, $R_{\mathrm{OO}}=2.79 \AA$, estimated as described in section VII, is consistent with the overall trend in the experimentally measured values as a function of cluster size (see Figure 2 of ref 1 ). This is consistent with the theoretical results of Xantheas, ${ }^{12}$ as well as others, which predict an exponential decrease in $R_{\mathrm{OO}}$ as a function of cluster size for cyclic clusters, approaching the ordered bulk limit by $n=4-6$.
Acknowledgment. This work was supported by the National Science Foundation under Grant CHE-9424482.

\section{References and Notes}

(1) Liu, K.; Cruzan, J. D.; Saykally, R. J. Science 1996, 271, 877.

(2) Elrod, M. J.; Saykally, R. J. Chem. Rev. (Washington, D.C.) 1994, 94, 1975.

(3) Dyke, T. R.; Mack, K. M.; Muenter, J. S. J. Chem. Phys. 1977, 66, 498. Busarow, K. L.; Cohen, R. C.; Blake, G. A.; Laughlin, K. B.; Lee, Y. T.; Saykally, R. J. J. Chem. Phys. 1989, 90, 3937. Coker, D. F.; Miller, R. E.; Watts, R. O.; J. Chem. Phys. 1985, 82, 3554. Hu, T. A. and Dyke, T. R. J. Chem. Phys. 1989, 91, 7348. Odutola, J. A.; Dyke, T. R. J. Chem. Phys. 1980, 72, 5062. Karyakin, E. N.; Fraser, G. T.; Suenram, R. D. Mol. Phys. 1993, 78, 1179. Karyakin, E. N.; Fraser, G. T.; Lovas, F. J.; Suenram, R. D.; Fujitake, M. J. Chem. Phys. 1994, 102, 114. Martinache, L.; JansBurli, S.; Vogelsanger, B.; Kresa, W., Bauder, A. Chem. Phys. Lett. 1988, 149, 424. Zwart, E.; ter Muelen, J. J.; Meerts, W. L. Chem. Phys. Lett. 1990, 173, 115. Zwart, E.; ter Muelen, J. J.; Meerts, W. L.; Coudert, L. H. J. Mol. Spectrosc. 1991, 147, 27. Pugliano, N.; Cruzan, J. D.; Loeser, J. G.; Saykally, R. J. J. Chem. Phys. 1993, 98, 6600.

(4) Cruzan, J. D.; Viant, M. R.; Liu, K.; Brown, M. G.; Braly, L. B.; Lucas, D. D.; Saykally, R. J. 1997, in preparation. 17.

(5) Huisken, F.; Kaloudis, M.; Kulcke, A. J. Chem. Phys. 1996, 104,

(6) Liu, K.; Loeser, J. G.; Elrod, M. J.; Host, B. C.; Rzepiela, J. A.; Saykally, R. J. J. Am. Chem. Soc. 1994, 116, 3507.

(7) Liu, K.; Brown, M. G.; Viant, M. R.; Cruzan, J. D.; Saykally, R. J. Mol. Phys. 1996, 89, 1373.

(8) Pugliano, N.; Saykally, R. J. Science 1992, 257, 1937.

(9) Suzuki, S.; Blake, G. A. Chem. Phys. Lett. 1994, 229, 499.

(10) Viant, M. R.; Cruzan, J. D.; Lucas, D. D.; Brown, M. G.; Liu, K.; Saykally, R. J. J. Phys. Chem. A 1997, 101, 9032.

(11) Viant, M. R.; Cruzan, J. D.; Brown, M. G.; Saykally, R. J. 1997, in preparation.

(12) Xantheas, S. S.; Dunning, T. H., Jr. J. Chem. Phys. 1993, 99, 8774

(13) van Duijneveldt van de Rijdt, J. G. C. M.; van Duijneveldt, F. B. Chem. Phys. 1993, 175, 271.

(14) Fowler, J. E.; Schaefer, H. F. J. Am. Chem. Soc. 1995, 117, 446

(15) Clementi, E.; Kolos, W.; Lie, G. C.; Ranghino, G. Int. J. Quantum Chem. 1980, 27, 377.

(16) Mó, O.; Yáñez, M.; Elguero, J. J. Chem. Phys. 1992, 97, 6628

(17) Xantheas, S. S.; Dunning, T. H., Jr. J. Chem. Phys. 1993, 98, 8037.

(18) Xantheas, S. S. J. Chem. Phys. 1994, 100, 7523.

(19) Xantheas, S. S. J. Chem. Phys. 1995, 102, 4505.

(20) Gregory, J. K.; Clary, D. C. J. Chem. Phys. 1995, 103, 8924.

(21) Gregory, J. K.; Clary, D. C. J. Phys. Chem. 1996, 100, 18014.

(22) Chałasiński, G.; Szczȩśniak, M. M.; Cieplak, P.; Scheiner, S. J. Chem. Phys. 1991, 94, 2873.

(23) Szczęśniak, M. M.; Chałasiński, G. J. Mol. Struct. (THEOCHEM) 1992, 261, 37.

(24) Franks, F., Ed. WATER, A Comprehensive Treatise; Plenum Press: New York, 1976.

(25) Belford, D.; Campbell, G. J. Chem. Phys. 1987, 86, 7013.

(26) Corongiu, G.; Clementi, E. J. Chem. Phys. 1993, 98, 2241.

(27) Dietrich, J.; Corongiu, G.; Clementi, E. Chem. Phys. Lett. 1984 $112,426$.

(28) Wojcik, M.; Clementi, E. J. Chem. Phys. 1986, 84, 5970.

(29) Corongiu, G.; Clementi, E. J. Chem. Phys. 1992, 97, 2030.

(30) Corongiu, G.; Clementi, E. Chem. Phys. Lett. 1993, 214, 367.

(31) Kim, K. S.; Dupuis, M.; Lie, G. C.; Clementi, E. Chem. Phys. Lett. 1986, $131,451$.

(32) Mhin, B. J.; Kim, J.; Lee, S.; Lee, J. Y.; Kim, K. S. J. Chem. Phys. 1994, $100,4484$.

(33) Mihn, B. J.; Kim, H. S.; Yoon, C. W.; Kim, K. S. Chem. Phys. Lett. 1991, 176, 41 .

(34) Ohmine, I.; Tanaka, H. Chem. Rev. (Washington, D.C.) 1993, 93, 2545.

(35) Lentz, B. R.; Scheraga, H. A. J. Chem. Phys. 1973, 58, 5926.

(36) Owicki, J. C.; Shipman, L. L.; Scheraga, H. A. J. Phys. Chem. 1975, 79, 1794.

(37) Dykstra., C. E. J. Chem. Phys. 1989, 91, 6472.

(38) Schütz, M.; Klopper, W.; Lüthi, H.-P. J. Chem. Phys. 1995, 103, 6114.

(39) Lee, C.; Chen, H.; Fitzgerald, G. J. Chem. Phys. 1995, 102, 1266 (40) Koehler, J. E. H.; Saenger, W.; Lesyng, B. J. Comput. Chem. 1987, 8,1090 .

(41) Head-Gordon, T.; Head-Gordon, M. 1995

(42) Laasonen, K.; Parrinello, M.; Car, R.; Lee, C.; Vanderbilt, D. Chem. Phys. Lett. 1993, 207, 208.

(43) Estrin, D. A.; Paglieri, L.; Corungiu, G.; Clementi, E. J. Phys. Chem. 1996, 100,8701 .

(44) Saykally, R. J.; Blake, G. A. Science 1993, 259, 1570. 
(45) Cruzan, J. D.; Braly, L. B.; Liu, K.; Brown, M. G.; Loeser, J. G.; Saykally, R. J. Science 1996, 271, 59.

(46) Cruzan, J. D.; Brown, M. G.; Liu, K.; Braly, L. B.; Saykally, R. J. J. Chem. Phys. 1996, 105, 6634.

(47) Liu, K.; Brown, M. G.; Cruzan, J. D.; Saykally, R. J. Science 1996, 271,62 .

(48) Cruzan, J. D.; Viant, M. R.; Lucas, D. D.; Saykally, R. J. 1997, work in progress.

(49) Liu, K.; Brown, M. G.; Gregory, J. K.; Carter, C.; Clary, D. C.; Saykally, R. J. Nature 1996, 381, 501.

(50) Schütz, M.; Bürgi, T.; Leutwyler, S. J. Chem. Phys. 1993, 99, 5228.

(51) Wales, D. J. J. Am. Chem. Soc. 1993, 115, 11180.

(52) Walsh, T. R.; Wales, D. J. J. Chem. Soc., Faraday Trans. 1996, $92,2505$.

(53) Gregory, J. K.; Clary, D. C. J. Chem. Phys. 1995, 102, 7817.

(54) Wales, D. J.; Walsh, T. R. J. Chem. Phys. 1996, 105, 6957.

(55) Gregory, J. K.; Clary, D. C. Chem. Phys. Lett. 1994, 228, 547.

(56) Gregory, J. K.; Wales, D. J.; Clary, D. C. J. Chem. Phys. 1994, 102,1592

(57) Gregory, J. K.; Clary, D. C. Chem. Phys. Lett. 1995, 237, 39

(58) Gregory, J. K.; Clary, D. C. J. Chem. Phys. 1996, 105, 6626.

(59) Townes, C. H.; Schawlow, A. L. Microwave Spectroscopy; Dover/McGraw-Hill: New York, 1975.

(60) Blake, G. A.; Laughlin, K. B.; Cohen, R. C.; Busarow, K. L.; Gwo, D.-H.; Schmuttenmaer, C. A.; Steyert, D. W.; Saykally, R. J. Rev. Sci. Instrum. 1991, 62, 1701.

(61) Liu, K.; Fellers, R. F.; Viant, M. R.; McLaughlin, R. P.; Brown, M. G.; Saykally, R. J. Rev. Sci. Instrum. 1996, 67, 410.

(62) Herriott, D. R.; Schülte, H. J. Appl. Opt. 1965, 4, 883.

(63) Kay, B. D.; Castleman, A. W., Jr. J. Chem. Phys. 1983, 78, 4297.
(64) Bunker, P. R. Molecular Symmetry and Spectroscopy, 1st ed.; Academic Press: San Diego, CA, 1979.

(65) Dyke, T. R. J. Chem. Phys. 1976, 66, 492.

(66) Brown, M. G.; Keutsch, F. 1996, private communication.

(67) Balasubramanian, K. J. Chem. Phys. 1980, 72, 665.

(68) van der Avoird, A.; Olthof, E. H. T.; Wormer, P. E. S. J. Chem. Phys. 1996, 105, 8034.

(69) Millot, C.; Stone, A. J. Mol. Phys. 1992, 77, 439.

(70) Astrand, P. O.; Linse, P.; Karlstrom, G. Chem. Phys. 1995, 191, 195.

(71) Stone, A. J. Chem. Phys. Lett. 1989, 155, 102.

(72) Axilrod, B. M.; Teller, E. J. Chem. Phys. 1943, 11, 299.

(73) Wales, D. J. J. Am. Chem. Soc. 1993, 115, 11191.

(74) Olthof, E. H. T.; van der Avoird, A.; Wormer, P. E. S. J. Chem. Phys. 1996, 105, 8051 .

(75) Fellers, R. F.; McLaughlin, R. P.; Viant, M. R. 1996, work in progress.

(76) Dyke, T. R.; Muenter, J. S. J. Chem. Phys. 1972, 57, 5011.

(77) Armbruster, M.; Haberland, H.; Schindler, H. G. Phys. Rev. Lett. 1981, 47, 323 .

(78) Haberland, H.; Ludewigt, C.; Schindler, H.; Worsnop, D. R. J. Chem. Phys. 1984, 81, 3742.

(79) Haberland, H.; Ludewigt, C.; Schindler, H.-G.; Worsnop, D. R. Surf. Sci. 1985, 156, 157.

(80) Garrett, W. J. Chem. Phys. 1979, 71, 651

(81) Kistenmacher, H.; Lie, G. C.; Popkie, H.; Clementi, E. J. Chem. Phys. 1974, 61, 546.

(82) Barnes, P.; Finney, J. L.; Nicholas, J. D.; Quinn, J. E. Nature 1979, 282, 459.

(83) Liu, K.; Brown, M. G.; Saykally, R. J. 1997, in preparation. 